\title{
Real-Time, Efficient e-Infrastructure Development Framework for Corporate Energy Sector
}

\author{
Jamshaid Iqbal Janjua and Zubair Ahmed Khan
}

\begin{abstract}
E-Infrastructure Development based on the local customized needs capitalized with the real time information gathering and decision making is an important \& challenging area for any nation to survive energy sector that acts as a backbone to nation's prosperity. We discussed the need for advancement in industrial infrastructures and services in energy sector of Pakistan. The proposed layer based framework provides the efficient replacement of the existing manual and out dated electronic system to automations. The developed subsystem is also discussed as a drive towards the change in the existing system. The proposed framework will provide a platform to all stakeholders to new levels of efficient and real-time corporate energy management.
\end{abstract}

Index Terms-Energy sector, e-infrastructure, real time, tariff indexation, power loss, facility performance, load forecasting, data acquisition, interoperability.

\section{INTRODUCTION}

Software is vital for modern society. It manages our finances, regulates communication and power generation, controls ground, marine and aerial traffics, and processes security-critical information. Consequently, the efficient development of dependable software is of every growing importance. Energy industry provides spine for a country's growth \& development and is one of the most important utilities that underpin survival of a nation's critical industrial infrastructures \& services.

Government of Pakistan announced a new policy for the Upcoming Power Generation Projects in 2002. This regime is approved by National Electric Power Regulatory Authority (NEPRA). To implement this regime a new standard Power Purchase Agreement (PPA) has been developed by National Transmission \& Dispatch Company (NTDC). Initially, there was a demand identified for an Efficient Data Collection, and Invoice Processing Automation System which supports the mandate of NTDC to prescribe the performance standards for efficient monitoring \& supply of electric power generation.

The new PPA of 2002 differs from its predecessor PPA of 1994 in its basic concept of financial transactions based on the Availability Based Billing. The main contractual entities are Independent Power Producers (IPPs) and NTDC as Power Purchaser (PP). Central Power Purchasing Agency (CPPA) \& System Operator (SO) are the sub companies of NTDC. CPPA is responsible for financial transactions between power generation and power distribution companies. It buys electricity form IPPs on behalf of NTDC and the NTDC sells this energy to power distribution companies.

Manuscript received December 14, 2012; revised February 25, 2013.

The authors are with Al-Khawarizmi Institute of Computer Science, University of Engineering \& Technology, Lahore, Pakistan (e-mail: jamshaid.janjua@kics.edu.pk, zubair.khan@uet.edu.pk).
Hence, CPPA acts as the Power Purchaser on Behalf of Government of Pakistan. The SO is responsible for secure, safe and reliable operation, control and dispatch of IPPs and other generation facilities.

The real time, efficient monitoring of the IPP and Grid generation facilities, results in the optimal use of the power generation capabilities and timely power energy availability to the business \& domestic users. This gives rise to the need for the definition of an electronic business infrastructure development model for the entities involved in energy supply and demand business in Pakistan, resulting in the replacement of the existing manual system or out dated electronic system and would assist the governing bodies to deal with the overall system deficiencies like institutional inability to carry out desired functions effectively[1], inappropriate planning, greater energy dependence and insecurity[2]. In such environment, high qualitative decision making is a challenging task in today's dynamic business environment [3].

This need makes a baseline for introducing automated enterprise grade software consortium that brings in a real-time, efficient, cost effective and transparent e-infrastructure framework to both WAPDA and the energy sector in Pakistan. This infrastructure is proposed as Real-time, Efficient, Corporate System (RECSys). Transparency refers to the vision of a glass tube though which the stakeholders can see the whole status of energy from generation to handing over to distribution side. Same time, the stakeholders can view the financial transactions from one end to other end i.e. IPP to Power Purchaser and vice versa, which mainly includes the Energy, Capacity Payments and Liquidated Damages \& Penalties to the IPPs. Another important aspect of the system is to focus the paperless paradigm in the long run for the whole process of buying and selling of the power generation facilities.

\section{FRAMEWORK}

RECSys relates with many technologies, techniques and tools that rise from the strategic information technology implementation policy of the government. The proposed framework in Fig. 1 has four levels of the information treatment. The overall proposed structure is of modular nature, as the sub-systems keep on coming in this framework, on the whole global enterprise solution transforms from offline to semi offline to real time environment moving to a deficient need of e-infrastructure development in Pakistan. Real time systems are becoming more and more important for successful business activity that requires data analysis to be performed in synchronized manner so that efficient \& real 
time monitoring with performance [4] can be made a base to predict future energy requirements.

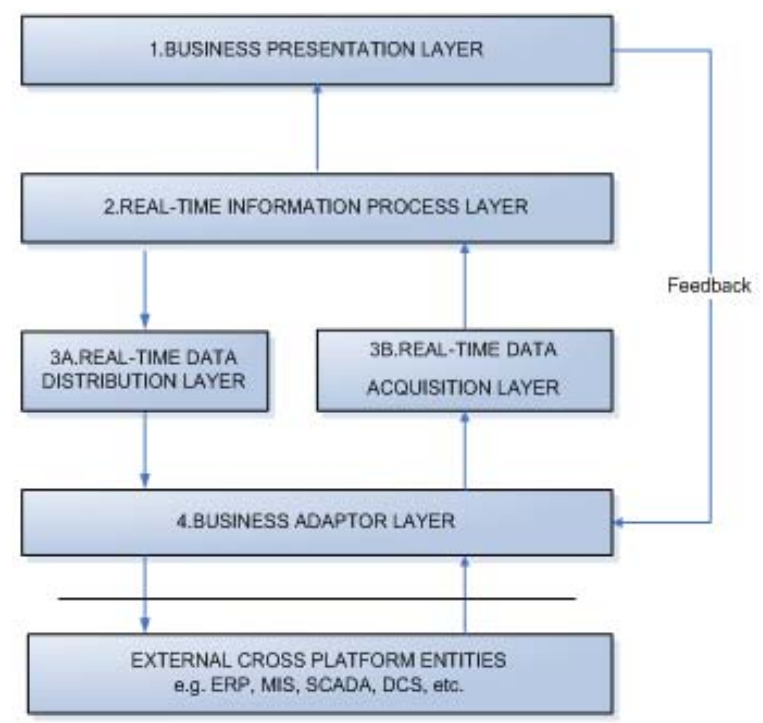

Fig. 1. RECSys layer based framework.

This framework is focused on the real time processing of the information and replacement of the existing manual system and the out dated electronic system to automations, in order provide a real time, efficient and cost effective information.

\section{ARCHITECTURE}

This section describes the architecture of the layer based proposed framework. The four layers are 1) Business Presentation Layer, 2) Real Time Information Process Layer, 3) Real Time Data Acquisition \& Distribution Layers, and 4) Business Adaptor Layer.

\section{A. Business Presentation Layer}

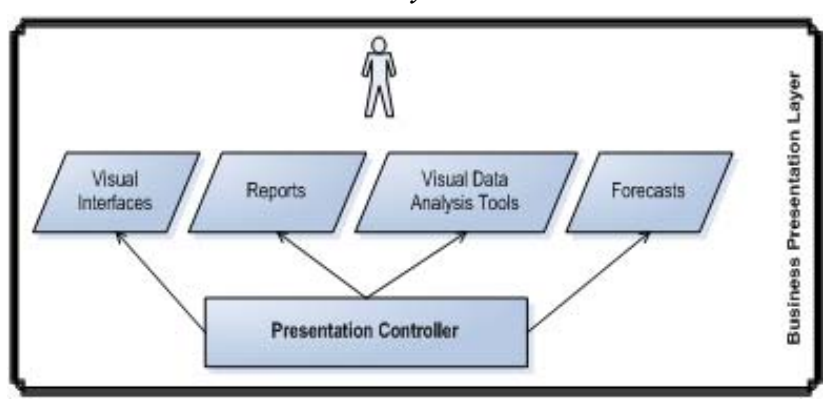

Fig. 2. RECSys business presentation layer 1.

This Layer 1 of proposed framework in Fig. 2 is responsible for the presentational visual interfaces including the web pages and various reports for different outcomes. This also includes the interactive graphical tools for information comparison and analysis. This assists both process managers and decision makers by the presentations of analysis based information in timely manner in overall energy management concerns. The visual objects are produced by the presentation controller, which takes the processed information from the Real-Time information process layer. This layer implements an overall system feedback process that enables the web services at the lowest layer to adapt in the standard framework protocols for external entities communication.

\section{B. Real-Time Information Process Layer}

The Layer 2 is responsible for the overall real time information processing. Due to the complex and diverse local customization needs of the energy sector in Pakistan, this layer is divided further into sub-sections so that the complexity of transforming and replacing the overall system from manual or out dated electronic system to global real time system can be achieved with minimum system conversion cost. The sub-sections include, 1) NEPRA Indexation Determination, 2) Invoice Processing \& Automation System, 3) Facility Performance \& Power Loss Determination, 4) Power Load Forecasting and 5) Merit Order Estimation for Generation Facilities.

The cardinal information source point for this layer is its Information Controller which provides the qualified and related data from either the repository database or from the Data Acquisition Controller in the lower layer 3 of the framework, later is this case mostly for the real-time information fetching. The processing relevant input data in purple of Fig. 3 is provided to the sub systems and the results in yellow are provided to the Presentation Controller of the Layer.1 for the presentation in different visual tools and formations as required by the user for information analysis and comparisons in real-time. The processed results are persisted in the database repository for the future utilization and reference.

\section{1) NEPRA indexation determination}

This subsystem deals with the calculation of the various tariff indexation adjustment factors time to time, so that the effects of factors like inflation, deflation, fuel indexes etc., are adjusted on the existing reference tariffs of the numerous generation facilities like IPPs, Common Delivery Points (CDPs), Public-sector Power Producers, Small Power Producers, etc.

The subsystem takes in relevant information from the Information Controller and processes it by applying the NEPRA business rules as prescribed in the PPA or NEPRA determinations to calculate adjustment factors for Consumer Price Index (CPI), Whole Price Index (WPI), Foreign and Local Loan Interest Rates references with LIBOR \& KIBOR adjustments, Fuel Cost Components for different reference tariffs. The relevant information sources may be predefined online sources. The calculated adjustment factors will be automatically published in online official gazette notifications and will be ready for use for different IPPs and other facilities in a more time-convenient way. The sub system will enable the overall enterprise solution to utilize the time important tariff indexation adjustments at the very applicable time. This reduces current system's overhead of reclaiming invoices and financial instruments again \& again for a single adjustment for being not available at exact applicable time. 


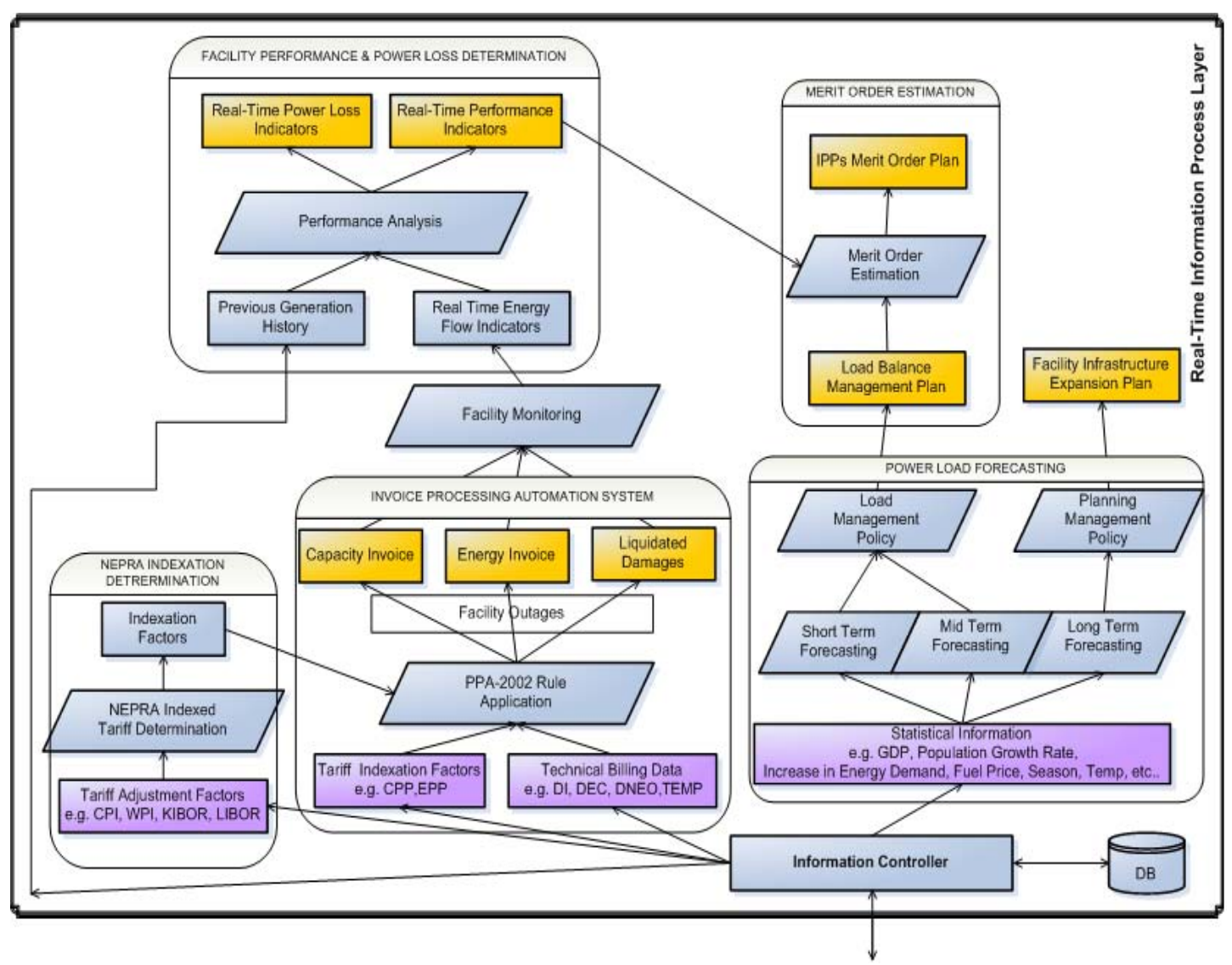

Fig. 3. RECSys real time information process layer 2 .

\section{2) Invoice processing \& automation system (IPAS)}

This sub system of Layer.2 provides a platform to energy governing body to have cleanest and transparent conduct of agreed terms and conditions with IPPs in PPA and efficient management of WAPDA's own energy resources. IPP's while following terms and conditions of the PPA, are required to declare their available generation capacity in advance, 12 months, 3 months, 14 days and 24 hours for medium term and short term available generation capacity. Followed by these commitments the IPPs are continuously providing the changes in available generation due to very abrupt environmental factors and unforeseen circumstances until energy is delivered in the specific operating hour. The System Operator observes this available generation capacity while giving the Dispatch Instruction to an IPP for efficient Load Balancing on the National Power Grid. Keeping in view this either side commitment the IPP delivers the net electrical output energy at ambient temperature, pressure and humidity levels under certain complex full or partial loading conditions with different fuel based energy generation units. The complex maintenance effects the availably declaration of IPP as per agreed terms and conditions in the PPA, which are covered in certain outages allowances for scheduled and non scheduled outages for an agreement year.

These factors affect the calculations of the financial instruments for the IPPs mainly such as Capacity Payments for administrative and operational costs, Energy Payments for the consumption of the fuel for energy generation and Liquidated Damages calculated for the consequence for IPPs for not fulfilling the either side commitment or not operating uptil the required performance level as agreed in the PPA.

This subsystem is one of the main information source providers in the proposed framework. We have developed this complete subsystem with all these preliminary outputs and interim outputs for its stakeholders. The subsystem outputs are persisted in the database repository as technical and financial data separately which will assist and provide cardinal relevant information to all other subsystems in this layer for the real-time facility monitoring, performance measurement, power loss calculation and power load forecasting.

The developed system is engineered and completed using the open source technologies \& tools (JSF, J2EE, MySql, etc.) as the main driver for the proposed framework while also keeping the solution cost effective for the stakeholders. We have processed multiple technical and financial data streams from diverse fuel based IPPs for more than six months and efficiently produced the results as per PPA indicating the issues and point of differences of the manual system considered as human error or manual handing complications of the PPA due to its half hourly based calculations.

\section{3) Facility performance \& power loss determination}

This subsystem is incorporated in the Layer.2 for the measure of the generation facility performance indexes. Another critical objective is to measure the power losses with respect to the independent generation facilities and clustered facilities like generation and distribution companies. This will also assist energy governing bodies in strengthening the continuous monitoring over mal practices, misuse or below standard operations of generation facilities resulting in optimal energy generation.

It takes the real time energy flow indicators as input from IPAS and the historical information of a generation facility or 
the cluster of facilities to calculate the power losses from different comparisons of the energy import and export at various interconnections end points of the facilities. This provides the location identification feature by the system of the energy generation facility that has a non acceptable power loss index. This assists in an efficient fault identification system in the energy sector.

Real time monitoring and performance is measured with the performance indexes, like Generation Availability Index, Compliance Index, Outages Index, Local Forecasting Index, etc. Multiple visual graphical tools can be presented at the Layer.1 of the framework. This information can further be used to increase the efficiency of the overall power load forecasting system in this layer and its outcomes.

\section{4) Power load forecasting}

This subsystem is responsible for power load forecasting for different time ranges. In order to accomplish PPA's terms and conditions, load forecasting emerged as a natural requirement for IPP's and there is a direct need at NTDC for such a system. In order to meet this requirement an intelligent software system for Pakistan based on energy governing body's business requirements is proposed in this subsystem of Layer.2. This would assist NTDC mainly in making precise unit commitment with IPP's and decision making operation of load flow distribution. It will facilitate NTDC to manage power flows, based on metering data collected from IPP's through the IPAS subsystem, local meteorological information and water flow conditions. This system can be used to predict future requirements of energy and forecasting load for unit commitment in the both Short term and Long term to ensure economical operations at NTDC.

Precise load forecasting helps an electric utility in making accurate unit commitment decisions, reduce spinning reserve capacity and schedule proper maintenance plan. Besides playing a key role in reducing the generation cost, it has a vital impact on reliability of power systems. The system operators use the load forecasting result as a basis of off-line grid network analysis to determine if the system might be vulnerable. If so, corrective actions should be prepared, such as load shedding, power purchases and bringing peaking units on line. A precise power short term load forecasting will result in economic cost saving and improving security operation condition [5]. Therefore Load Forecasting has critical importance for the electric industry in a deregulated economy.

This subsystem will get statistical information like energy supply and demand information, temperature, seasons, events, fuel prices, etc. from Information Controller accompanied by the real-time facility performance indicators and other relevant information to predict the short, medium and long term energy projected requirement. Other factors are country's region based economical, environmental and social factors that are different from other countries, which affect on the demand, supply and generation of electrical energy. Different forecasting techniques can be adapted like Artificial Neural Networks, Regression, Time Series, etc. to match with the requirement. Local subsystem feedback mechanism improves the system prediction training over time. The outcomes of short term and medium term forecasting would generate the Load Balance Management
Plan that will help NTDC for its energy power flows and distribution management. The outcome of the long term forecasting would be Generation Facility Infrastructure Expansion Plan that will assist the energy sector governing bodies to plan for power infrastructure development \& expansion in Pakistan, keeping in view the infrastructure reserves for impromptu and downfall situations while maintaining a balance in demand and supply of energy generation for business and domestic concerns.

\section{5) Merit order estimation}

This subsystem bases on Facility Performance \& Power Loss Determination and Power Load Forecasting subsystems in Layer.2. In order to efficiently managing all the generation facilities, it is a very vital decision for the Energy Purchaser to decide how much business to give to a specific Power Producer. This involves many factors like performance, compliance, availability, complex life, fuel, etc in making this decision called merit order listing for next year business call for a power generation facility.

An important factor to join in this decision is the outcomes of the short term and medium term power load forecasting under the Load Management Policy. Along with the multiple previously mentioned factors the merit order estimation would handle the local generation trends of the generation facilities helping the energy purchaser to make a beneficial merit order that facilitates the business interest at either sides and helping NTDC to reach to a new height of smooth energy management in Pakistan.

\section{Real-Time Data Layer}

This Layer.3 is divided into two sub layers, 1) Real-Time Data Acquisition Layer and 2) Real-Time Data Distribution Layer. These both sub layers have independent data controllers for the efficient real time framework load management avoiding the coupling in the system.

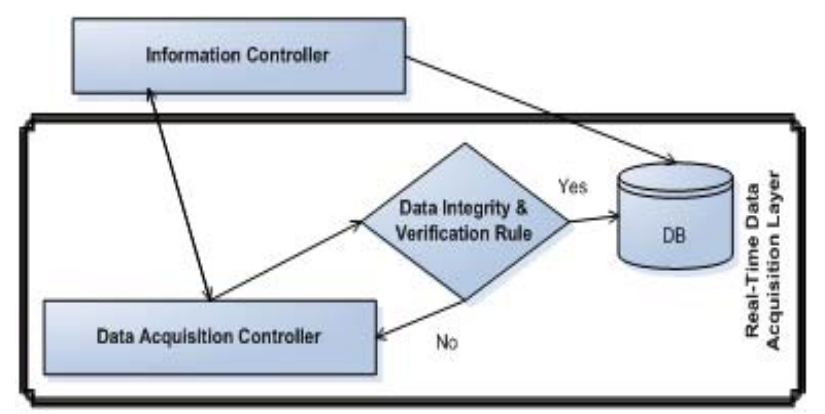

Fig. 4. RECSys real-time data acquisition layer 3A

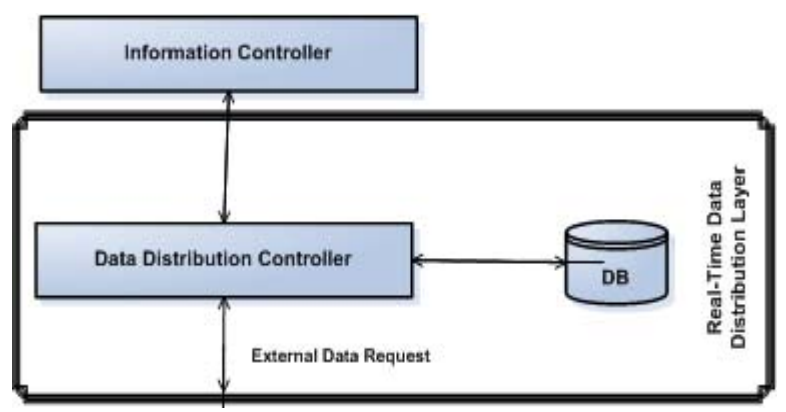

Fig. 5. RECSys real-time data distribution layer 3B

\section{1) Real-time data acquisition layer}

This sub layer in Fig. 4 is responsible for the Data 
Acquisition for the entire corporate solution. The controller in this layer firstly, provides any real time data request to the Information Controller in upper layer after fetching this information from the required external source through the lower layer. Secondly, the controller is responsible for the Data Integrity Checks and Data Verification, the passed data may be inserted in the database repository, and the else is to re-fetch the information from different remote software entities like SCADA, MIS, ERPs, DCS, etc. and hardware entities like Energy Meters, Weather Stations, Generation Units, etc.

\section{2) Real-time data distribution layer}

This sub layer Fig. 5 is responsible for the Data Distribution to any external entity data request in standard framework protocols. This sub layer fetches data from repository database prepares the data in specific format according to the protocols and dispatches the information in response to the exterior requests through its lower layer to external software entities like SCADA, MIS, ERPs, DCS, etc.

\section{Business Adaptor Layer}

Enterprise information systems must be able to adapt the environmental changes quickly and flexibly. Enterprises control large amounts of software resources and these software resources must be integrated [6] in order to form interoperability possible. The same can be extended towards the multiple vendor hardware resources at a single platform.

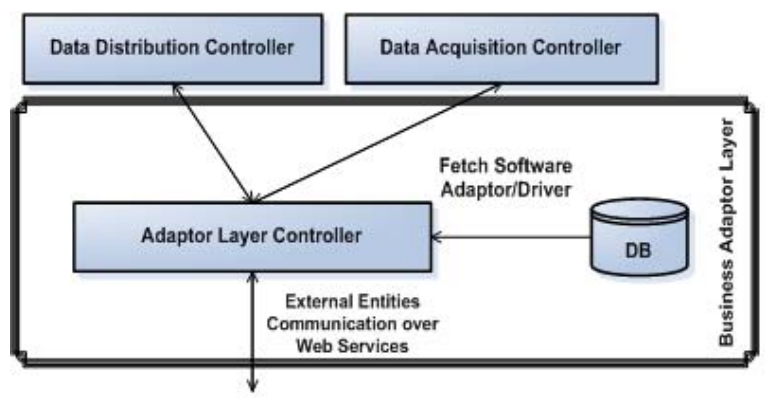

Fig. 6. RECSys business adaptor layer 4.

This is underline layer in Fig. 6 for whole framework; it provides an interface to the framework to communicate with the rest of the world in terms of electronic data exchange. This is in terms of communicating in interoperable manner with the cross platform software and hardware. This layer handles with the hardware drivers of the different hardware vendors for energy meters, weather stations, generation units, etc and acts as an adaptor to all the system calls from the Data Acquisition Controller and Data Distribution Controller from the upper layer. For all external software systems like SCADA, MIS, ERPs, DCS, etc, this layer provides Web Services based on Service Oriented Architecture (SOA) that assists implementing the standard framework protocols.

\section{APPLICATIONS}

The proposed RECSys system architecture can be applied in the Pakistan energy sector enabling it to cope with the local challenges to the country and the deregulated economy. It can assist the energy governing body in Pakistan to establish an
e-Infrastructure that is customized to local needs and can act as national requirement compliance for optimizing the working business model in energy sector as a start.

Power industry around the globe has developed their own energy management \& load forecasting systems to meet demand and supply requirements of energy where as there is no such system in place in Pakistan. Therefore it is a matter of critical need for both energy governing bodies and IPP's to have a real-time energy efficient management system. It can assist Pakistan in many energy related areas such as energy billing and invoicing, performance measurement, power loss estimation, purchasing and generation, load switching \& balancing, contract evaluation, and infrastructure development, etc.

This replacement of the existing manual and out dated electronic system initially, may be carried out on stabilized energy generation facilities, so that the minimum change effect is caused. After stabilization of new energy generation nodes in the system, further nodes may be introduced. The process continues for all facilities to join national energy electronic consortium.

\section{CONCLUSION}

Power sector plays a cardinal role in the country's growth and development in this era of need for nation's advancement in industrial infrastructures and services in line with the modern and advanced countries. A customizable solution is required to uplift the country to new levels of management and decision making that brings in the stability and consistency to the national decisions in energy sector. The proposed framework RECSys assists the overall system to operate at an improved level of efficiency, accompanied with the real-time information acquisition and its analysis, comparisons in order to equip the energy governing bodies with the information required to make the correct, efficient, effective and long term decisions. We proposed generic framework architecture and its applications in the energy sector of Pakistan.

\section{ACKNOWLEDGMENT}

We are grateful to Al-Khawarizmi Institute of Computer Science, University of Engineering \& Technology, Lahore, Pakistan, for providing us the platform to work on the Invoice Processing \& Automation System (IPAS) that made us to propose RECSys framework. We also acknowledge WAPDA, Pakistan for funding that helped us to complete the initiative.

\section{REFERENCES}

[1] M. Afia, "Regulatory Governance: Electricity Industry in Pakistan," International Journal of Regulation and Governance, 2007, vol. 7, no. 1, ISSN: 0972-4907.

[2] A. Ghafoor and W. John, "Privatisation of Electric Power Sector in Pakistan: Some Important Issues," The Pakistan Development Review, vol. 38, no. 1, pp. 69-84, 1999.

[3] S. Asghar, S. Fong, and T. Hussain, "Business Intelligence Modeling: A Case Study of Disaster Management Organization in Pakistan," in Proc. The 4th International Conference on Computer Sciences and Convergence Information Technology (ICCIT 2009), 24-26 November 2009, Seoul, Korea, pp. 637-638.

[4] B. Azvine, Z. Cui, D. D. Nauck, and B. Majeed, "Real time business intelligence for the adaptive enterprise," in Proc. The 8th IEEE International Conference on E-Commerce Technology and The 3rd IEEE international Conference on Enterprise Computing, 
E-Commerce, and E-Services, Washington, DC, June 26 - 29, 2006, pp. 29.

[5] W. J. Dai and P. Wang, "Application of pattern recognition and artificial neural network to load forecasting in electric power system," in Proc. Third International Conference on Natural Computation (ICNC 2007), vol. 1, pp. 381-385, 2007.

[6] M. Oba and N. Komoda, "Multiple type workflow model for enterprise application integration," in Proc. 34th Annual Hawaii International Conference on System Sciences (HICSS-34), vol. 7, pp. 7048, 2001.

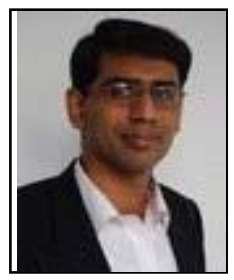

Jamshaid Iqbal Janjua received the MCS degree in computer science from Institute of Management Sciences, Lahore, Pakistan, in 2002. He earned the MBA degree as well from the same institute in 2007. He established the MS degree in Computer Science in 2009 at Faculty of Information Technology, University Central Punjab, Lahore, Pakistan.

The author became a Member of IEEE in 2011. He is an active member of IEEE Computer Society, Lahore Section. He is Senior Manager Technical Research at with Al-Khawarizmi Institute of Computer Science (KICS), University of Engineering \& Technology, Lahore, Pakistan. His research interests are Business Intelligence, E-Infrastructure Development, Forecasting \& Stochastic Processes, Software Design \& Architecture, Enterprise Grade
Energy Management Systems \& Surveillance Science.

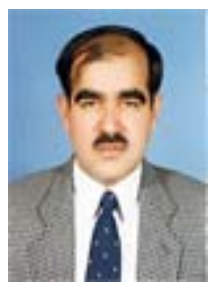

Zubair Ahmed Khan received the B.Sc. degree in electrical engineering from University of Engineering \& Technology, Lahore, Pakistan in 1975. He has the M.Sc. Electrical Engineering from same university in 1985. He earned his MS degree in electrical engineering from UMIST, Manchester, England in 1987 followed by his Ph.D. in electrical engineering in 1990 from UMIST, Manchester, England.

$\mathrm{He}$ is Dean Electrical Engineering at University of Engineering \& Technology, Lahore, Pakistan. His research interests are Microprocessor/ microcontroller/DSP processors \& FPGA/CPLD based system development and designing, Remote Metering/Data Acquisition, Energy Management Systems, E-Infrastructure Development and Enterprise Management Information Systems. 